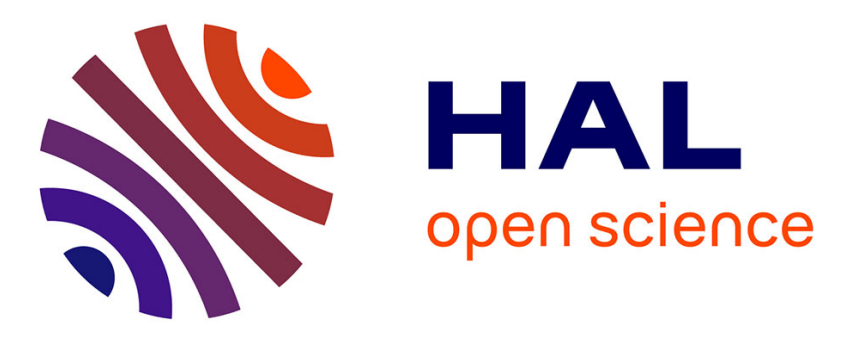

\title{
In-line monitoring of the primary drying phase of the freeze-drying process in vial by means of a Kalman filter based observer
}

\author{
Salvatore Velardi, Hassan Hammouri, Antonello A. Barresi
}

\section{To cite this version:}

Salvatore Velardi, Hassan Hammouri, Antonello A. Barresi. In-line monitoring of the primary drying phase of the freeze-drying process in vial by means of a Kalman filter based observer. Chemical Engineering Research and Design, 2009, 87 (10), pp.1409-1419. 10.1016/j.cherd.2009.03.011 . hal00433547

\section{HAL Id: hal-00433547 https://hal.science/hal-00433547}

Submitted on 11 Oct 2010

HAL is a multi-disciplinary open access archive for the deposit and dissemination of scientific research documents, whether they are published or not. The documents may come from teaching and research institutions in France or abroad, or from public or private research centers.
L'archive ouverte pluridisciplinaire HAL, est destinée au dépôt et à la diffusion de documents scientifiques de niveau recherche, publiés ou non, émanant des établissements d'enseignement et de recherche français ou étrangers, des laboratoires publics ou privés. 
This document must be cited according to its final version which is published in a journal as:

S. Velardi ${ }^{1}$, H. Hammouri ${ }^{2}$, A. Barresi ${ }^{1}$

"In-line monitoring of the primary drying phase of the freeze-drying process in vial by means of a Kalman filter based observer", Chemical Engineering Research \& Design 87,10 (2009) 1409-1419

This final version may be found: http://dx.doi.org/10.1016/j.cherd.2009.03.011

All open archive documents of $\mathbf{H}$. Hammouri are available at: http://hal.archives-ouvertes.fr/HAMMOURI-HASSAN

All open archive documents of $\mathbf{H}$. Hammouri research group (SNLEP) are available at: http://hal.archives-ouvertes.fr/SNELP http://www.tinyurl.com/SNELP

The professional web page (Fr/En) of $\mathrm{H}$. Hammouri is:

http://www.lagep.univ-lyon1.fr/signatures/hammouri.hassan

1 POLITO Torino Italy

Dip. di Scienza dei Materiali ed Ingegneria Chimica, Politecnico di Torino, 10129 Torino, Italy http://www.dismic.polito.it

2

Université de Lyon, Lyon, F-69003, France; Université Lyon 1;

CNRS UMR 5007 LAGEP (Laboratoire d'Automatique et de GEnie des Procédés),

43 bd du 11 novembre, 69100 Villeurbanne, France

Tel +33 (0) 472431845 - Fax +33 (0) 472431699

http://www-lagep.univ-lyon1.fr/ http://www.univ-lyon1.fr http://www.cnrs.fr 
accepted for the publication on Chem Eng Res Des (with minor revision)

CHERD-D-08-00462 Revised version 1 (March 2009)

\section{In-line monitoring of the primary drying phase of the freeze-drying process in vial by means of a Kalman filter based observer}

Salvatore A. Velardi ${ }^{1}$, Hassan Hammouri ${ }^{2}$, Antonello A. Barresi ${ }^{1 *}$

1. Dipartimento di Scienza dei Materiali e Ingegneria Chimica, Politecnico di Torino, C.so Duca degli Abruzzi 24, Torino, 10129, Italy

2. Université de Lyon, F-69003, France; Université Lyon 1; CNRS UMR 5007 LAGEP ; 43 bd du 11 Novembre 1918, 69100 Villeurbanne, France.

\footnotetext{
${ }^{*}$ Corresponding author: prof. Antonello A. Barresi e-mail: antonello.barresi@polito.it Tel: +39-011-5644658 Fax: +39-011-5644699
} 


\begin{abstract}
This paper is focused on the monitoring of the primary drying phase of the lyophilisation process of pharmaceuticals in vial. Monitoring is required to ensure that the maximum temperature of the product is maintained at a safe value in order to avoid denaturation, and the position of the moving front of sublimation has to be monitored since its evolution gives the state of progression of the primary drying. Furthermore, the information coming from the monitoring system, which include the estimation of the transport coefficients, can be used in a control loop designed to minimise the drying time beside ensuring product quality.

To this purpose, a soft-sensors (observer) has been developed, based on the extended Kalman filter algorithm: it requires a model of the process (a simplified model is used in order to reduce the computational load) and some physical measurements (in this case the temperature of the product at the bottom of the vial, that can be measured by a thermocouple). The main issues arising in the design of this observer have been discussed. A detailed mono-dimensional model experimentally validated has been used at first to compare the results provided by the observer by means of numerical simulations, and then the results obtained in a pilot freeze-dryer are shown.
\end{abstract}

\title{
Keywords
}

- Freeze-drying

- Lyophilization

- Primary drying

- Modelling

- Monitoring

- Extended Kalman filter 


\section{Introduction}

Freeze-drying, or lyophilisation, is the process where water or another solvent is removed from a frozen product by sublimation. The lyophilization technique consists of three main steps:

1. Freezing: the product to be dried is frozen at low temperature;

2. Primary drying: in this phase the ice is sublimated, generally operating under reduced pressure. Vapour originated at the moving sublimation front flows through the dried material into the lyophilization chamber and a refrigerated trap connected to the chamber - the ice condenser - continuously removes it. In vial freeze-drying heat is continuously supplied to the product through an heating shelf; this is required in order to compensate for the energy required by the endothermic sublimation process.

3. Secondary drying: the last stage of the freeze-drying process is a desorption step where the residual moisture, which is strongly bounded by adsorption phenomena to the partially dried cake, is reduced to a low level ensuring long term product preservation at room temperature. This step is usually carried out at high vacuum and moderate temperature $\left(+20\right.$ to $\left.+60^{\circ} \mathrm{C}\right)$.

In most of the industrial freeze-dryers control actions are often based on empirical information obtained in previous experimental runs carried out with the product of interest. Nevertheless, Guidance for Industry PAT (Process Analytical Technology) issued by the US Food and Drug Administration in September 2004, encourage to develop in-line monitoring and control tools in order to improve the manufacturing process. The most important parameter to be monitored and to be controlled during primary drying is the temperature of the product. In fact primary drying should be carried on at a temperature below the eutectic point of the crystalline solute, to avoid formation of liquid. Many solutes, such as proteins, do not crystallise during freezing, remaining amorphous. The formation of a glass can be beneficial for the protein activity preservation; but, it makes the drying process more demanding in terms of process time and physical conditions, because the primary drying must be carried out below the glass transition temperature to avoid collapse of the cake structure. Another important variable that would be useful to monitor is the position of the sublimating interface, the evolution of which gives the state of progression of the primary drying. Mass transfer across the porous matrix of the product and heat transfer between the shelf and the vial 
are two other critical aspects that influence the drying time. Mass transfer is related to the temperature and pressure conditions and to the resistance of the dried cake to the vapour flow. Heat transfer is mainly linked to the composition and pressure of the gas phase in the lyophilisation chamber and to the geometry of the vial in the region of contact with the heating shelf.

The insertion of thin thermocouples in the vials is a widely used method to measure the product temperature. Moreover, if multiple thermocouples are inserted the position of the moving front can be monitored. In fact, as the sublimating interface passes in correspondence of the sensor, a change in the slope of the temperature profile is observed since the thermal conductivity of the frozen and dried layer is different. However, for practical reasons, only one thermocouple is generally used placed in close contact with the internal bottom of the vial, whose temperature is assumed representative of the whole product. The method proposed in this paper, based on the Extended Kalman Filter (EKF) concept, is able to determine the temperature of the product at any axial position, as well as the dynamic evolution of the sublimating interface, only exploiting the single point measure of the temperature at the bottom of the product. Some parameters related to mass and heat transfer are also estimated, that can be fed to an in-line predictive control system (Fissore et al., 2008). Furthermore, differently from manometric temperature measurement (MTM) like methods (for a review, see Velardi et al., 2008) , this approach allows continuous monitoring either in one or several single vials placed in different positions, giving a direct in-line measure of the batch variance.

Most of the modern theories on the control of dynamic systems are actually based on a state-space representation. This representation allows for the description of the system behaviour through the variation of its state. The state of a system can be defined as the minimum set of information needed to completely describe the system at a given time instant. The knowledge of the vector of the state is needed in order to apply the command law. This can be achieved by using physical sensors. However, in many cases due to cost consideration and physical constraints, the number and type of sensors could be very limited. An observer, or soft-sensor, combines a priori knowledge about the physical system - the model - with experimental data - the on-line measurements to provide an on-line estimation of states and/or parameters.

The synthesis of observers for non-linear systems is generally a difficult task and 
many types of observers have been proposed in the literature. All the approaches proposed are based on the analysis of the observability (sensitivity of the output measurements with respect to state). The observability analysis yields to special forms of systems (called normal form, or canonical form). Among these canonical forms, linear systems up to output injection are systems whose dynamics can be splitted into two parts: a linear one with respect to state and a nonlinear one which depends only on the output measurements (Krener and Isidori, 1983). For these systems, it is possible to design a Luenberger observer. Using a Kalman-like observer, this result has been extended to state affine systems up to output injection (Hammouri and Gauthier, 1992).

Kalman Filter also requires some observability hypothesis in order to guaranty the stability of the filter. For the time-variant linear systems Kalman and Bucy (1961) showed that if the system is completely uniformly observable then the filter becomes stable. Similar hypothesis can be assumed in order to design an extended Kalman observer. A peculiarity of the Extended Kalman Filters is that they can be used without transforming the system on a particular form. However, the convergence is guaranteed only if the initialization of the observer is chosen in a local domain of the unknown state. In practice this domain may be large. The calibration of the constant matrix gain which appears in the Riccati equation depends on the dimension of the system and the output sensitivity. For small dimension and in the single output case, it is generally easy to obtain this calibration.

The EKF has been chosen for this work as it is well known in the process industries and the most frequently used state estimator (Cutler et al., 1983; Richalet, 1993; Qin and Badgwell, 1997). In spite of its use, relatively few papers have been published on industrial application of Kalman filtering: some examples can be found in the works by Dimitratos et al. (1991), Krämer et al. (2003), Alvares and Simutis (2004), Bentes Freire and Giudici (2004). In the authors knowledge this is the first published application referring to freeze-drying of pharmaceuticals.

The structure of the paper is the following: in the first section the simplified model used to design the EKF is introduced; then, the observer is given and the results obtained are discussed. Basic concepts concerning the EKF and details on the calculations are summarised in Appendix A and Appendix B. 


\section{The simplified model}

The simplified model that is used to design the EKF was previously shown by Velardi (2004) and Velardi and Barresi (2008). It is based on the energy balance for the frozen product and the mass balance for the water vapour inside the dried product, both taken in pseudo-stationary conditions because of the slow dynamics of the process. The presence of an inert gas is neglected as well as heat transfer in the vial glass. The temperature at the bottom of the product is assumed to be the physically measured variable of the process. The reference geometry is shown in Figure 1. The final form of the simplified mono-dimensional model is given by:

$$
\begin{aligned}
& \frac{d H}{d t}=\frac{1}{\varrho_{I I}-\varrho_{I e}} \frac{M}{R T_{i}} \frac{k_{1}}{H}\left(p_{w, i}\left(T_{i}\right)-p_{w, c}\right) \\
& \left(\frac{1}{K_{v}}+\frac{L-H}{k_{I I}}\right)^{-1}\left(T_{\text {shelf }}-T_{i}\right)=\frac{\Delta H_{s} M}{R T_{i}} \frac{k_{1}}{H}\left(p_{w, i}\left(T_{i}\right)-p_{w, c}\right) \\
& T_{I I}(z)=T_{\text {shelf }}-\frac{1}{K_{v}}\left(\frac{1}{K_{v}}+\frac{z-H}{k_{I I}}\right)^{-1}\left(T_{\text {shelf }}-T_{i}\right)
\end{aligned}
$$

The key parameters of the model are the mass transfer coefficient in the dried layer $\left(k_{1}\right)$ and the heat transfer coefficient between the shelf and the bottom of the product $\left(K_{v}\right)$. The non-linear equation (2) provides a relationship between the interface temperature, $T_{i}$, and the position of the interface between the frozen and the dried region, $H$, while Eq. (3) gives the temperature profile of the product along the axial coordinate $z$. Thus, the value of the product temperature at the bottom of the sample $\left(T_{B}\right)$, that is the measured variable of the process, can be determined setting $z=L$ in Eq. (3). Eq. (1) describes the dynamics of the interface, relating it to the sublimation flux.

The proposed model is a quite simplified one, since heat transfer in the dried layer is not considered and pseudo-steady state is assumed in the frozen layer, leading to the approximation that all the energy transferred to the product is used for sublimation. Furthermore the vial sides are assumed to be insulated from radiation and this makes this model not suitable for the vials located near the edge of the tray or in proximity of the walls of the chamber.

The adequacy of this model to describe the dynamics of the primary drying of a freeze-drying process has been proven by means of comparison with the results 
obtained using a detailed one-dimensional model experimentally validated (Velardi and Barresi, 2008).

\section{Kalman filter design and validation}

In this section an Extended Kalman Filter type observer is synthesised using the simplified model previously developed. The target observer should estimate the dynamics of the interface temperature at any time during primary drying, by using the temperature at the bottom of the frozen product as measured variable. The effective diffusion coefficient in the dried layer, that affects mass transfer, and the heat transfer coefficient between the shelf and the vial bottom, are considered as unknown parameters and are estimated too.

In order to develop an observer with the required structure [that will be shown by Eq. (19)-(22)], the system must be put in the general state-based form:

$\dot{\mathbf{x}}=\mathbf{f}(\mathbf{x}, u)+\mathbf{v}(t)$

$y=h(\mathbf{x}, u)+\mathbf{w}(t)$

where $u$ and $y$ are the scalar input and output of the system (4) respectively (because a single manipulated input and a single measure are adopted), and $\mathbf{v}(t)$ and $\mathbf{w}(t)$ are the additive noises on the state dynamics and on the output measurements. Details about the EKF and its convergence are summarised in Appendix A.

Since it is proposed to estimate the dynamics of the interface temperature $T_{i}$, and the values of the diffusion coefficient $k_{1}$ and of the heat transfer coefficient $K_{v}$, the state vector of the process can be defined as:

$\mathbf{x}=\left(\begin{array}{lll}x_{1} & x_{2} & x_{3}\end{array}\right)^{T}=\left(\begin{array}{lll}T_{i} & K_{v} & k_{1}\end{array}\right)^{T}$ and $u=T_{\text {shelf }}$

while the state equation of the measure, giving the frozen product bottom temperature $T_{B}$, can be expressed as:

$y=h\left(x_{1}, x_{2}, x_{3}, u\right)=T_{\mathrm{B}}\left(T_{i}, K_{v}, k_{1}, T_{\text {shelf }}\right)$

where the shelf temperature $T_{\text {shelf }}$ is taken as process input. Thus, the dynamic system to be solved can be written as: 
$\dot{\mathbf{x}}=\left(\begin{array}{c}\dot{x}_{1} \\ \dot{x}_{2} \\ \dot{x}_{3}\end{array}\right)=\left(\begin{array}{l}\frac{d x_{1}}{d t} \\ \frac{d x_{2}}{d t} \\ \frac{d x_{3}}{d t}\end{array}\right)=\left(\begin{array}{c}f_{1}\left(x_{1}, x_{2}, x_{3}, u\right) \\ 0 \\ 0\end{array}\right)=\mathbf{f}(\mathbf{x}, u)$

$y=h(\mathbf{x}, u)$

where the first derivatives of $x_{2}=K_{v}$ and $x_{3}=k_{1}$ are set equal to zero because they are considered constant parameters of the process. Of course this is an approximation, but it is correct if we assume that the observer can almost compensate for the variation of $K_{v}$ and $k_{1}$ through the effect of the term $\mathbf{K}(t)(\hat{y}-y)$ [see Eq. (19) in the followings], that corrects the dynamics of the state estimates $\dot{\hat{\mathbf{x}}}$. The dynamic equation (1) of the simplified model only provides the evolution of the position of the moving front $H$, but the form of system (8)-(9) requires an expression for the derivative of $x_{1}=T_{i}$ with respect to time. This can be calculated by differentiating with respect to time the function $H=H\left(T_{i}, K_{v}, k_{1}, T_{\text {shelf }}\right)=H(\mathbf{x}, u)$, obtaining:

$\frac{d x_{1}}{d t}=\left(\frac{d H}{d t}-\frac{\partial H}{\partial u} \frac{d u}{d t}\right)\left(\frac{\partial H}{\partial x_{1}}\right)^{-1}$

It follows from Eq. (10) that in order to determine the expression of $d x_{1} / d t$, the partial derivative of $H$ with respect to $x_{1}$ has to be calculated; thus the expression of $H$ as a function of the state is needed and it can be determined by rearranging equation (2), finding:

$$
H=\frac{\alpha \gamma}{\alpha+\beta}
$$

where the following substitutions have been made:

$$
\begin{aligned}
& \alpha=\alpha\left(x_{1}, x_{3}\right)=\frac{\Delta H_{s} M}{R} \frac{x_{3}}{x_{1}}\left(p_{w, i}\left(x_{1}\right)-p_{w, c}\right) \\
& \beta=\beta\left(x_{1}, u\right)=k_{I I}\left(u-x_{1}\right) \\
& \gamma=\gamma\left(x_{2}\right)=\frac{k_{I I}}{x_{2}}+L
\end{aligned}
$$

defining in addition:

$$
\delta=\frac{1}{\Delta H_{s}\left(\varrho_{I I}-\varrho_{I e}\right)}
$$


Adopting the substitutions (12)-(15), the expressions for $d H / d t, \partial H / \partial x_{1}$ and $\partial H / \partial u$ are obtained, the last one determined by derivation of Eq. (11):

$$
\begin{aligned}
& \frac{d H}{d t}=\delta \frac{\alpha+\beta}{\gamma} \\
& \frac{\partial H}{\partial u}=\frac{\gamma}{(\alpha+\beta)^{2}}\left(-\alpha \frac{d \beta}{d u}\right) \\
& \frac{\partial H}{\partial x_{1}}=\frac{\gamma}{(\alpha+\beta)^{2}}\left(\beta \frac{\partial \alpha}{\partial x_{1}}-\alpha \frac{d \beta}{d x_{1}}\right)
\end{aligned}
$$

The combination of the three previous equations according to Eq. (10), finally yields the dynamic evolution of the interface temperature $x_{1}=T_{i}$. Thus, with the previous notation the estimate state $\hat{x}=\left(\hat{x}_{1}, \hat{x}_{2}, \hat{x}_{3}\right)$ of $\left(x_{1}, x_{2}, x_{3}\right)$ is given by the EKF dynamic system:

$$
\begin{aligned}
& \dot{\hat{\mathbf{x}}}(t)=\left(\begin{array}{c}
\left.\left.\delta \frac{(\hat{\alpha}+\hat{\beta})^{3}}{\hat{\gamma}^{2}}+\hat{\alpha} \frac{d \hat{\beta}}{d u} \frac{d u}{d t}\right] \frac{1}{\left.\hat{\beta} \frac{\partial \hat{\alpha}}{\partial x_{1}}\right|_{\hat{\mathbf{x}}}-\left.\frac{d \hat{\beta}}{d x_{1}}\right|_{\hat{\mathbf{x}}} \hat{\alpha}}\right)-r \mathbf{K}(t)(\hat{y}(t)-y(t))= \\
0
\end{array}\right) \\
&= \mathbf{f}(\hat{\mathbf{x}}, u)-r \mathbf{K}(t)(\hat{y}(t)-y(t)) \\
& \hat{y}=u-\frac{\hat{\alpha}+\hat{\beta}}{\hat{x}_{2} \hat{\gamma}}=h(\hat{\mathbf{x}}, u) \\
& \mathbf{K}(t)=\mathbf{S}^{-1}(t)\left(\left.\frac{\partial h}{\partial \mathbf{x}}\right|_{\hat{\mathbf{x}}, u}\right)^{T} \\
& \dot{\mathbf{S}}(t)=-\mathbf{S}(t) \Lambda \mathbf{S}(t)-\left(\left.\frac{\partial \mathbf{f}}{\partial \mathbf{x}}\right|_{\hat{\mathbf{x}}, u}\right)^{T} \mathbf{S}(t)-\left.\mathbf{S}(t) \frac{\partial \mathbf{f}}{\partial \mathbf{x}}\right|_{\hat{\mathbf{x}}, u}+\left.r\left(\left.\frac{\partial \mathbf{h}}{\partial \mathbf{x}}\right|_{\hat{\mathbf{x}}, u}\right)^{T} \frac{\partial \mathbf{h}}{\partial \mathbf{x}}\right|_{\hat{\mathbf{x}}, u}
\end{aligned}
$$

where $\hat{\alpha}=\alpha\left(\hat{x}_{1}, \hat{x}_{3}\right), \hat{\beta}=\beta\left(\hat{x}_{1}, u\right), \hat{\gamma}=\gamma\left(\hat{x}_{2}\right)$ (see Eq. $(12,13,14)$ for these expressions), $y(t)$ is the on-line physical output measurement, $\Lambda$ is a symmetric positive definite matrix, and $r$ is a positive tuning parameter. Once the state of the system is known, the other variables of interest for process monitoring and control can be easily evaluated: in fact, the position of the interface, which gives the progress of the drying operation can be calculated by Eq. (11), while the sublimation flux can be obtained by the differentiation of the previous variable, or better from Eq. $(11,12)$, noting that it is simply given by $(\alpha+\beta) / \gamma \Delta H_{s}$ [compare also Eq. (1)]. Further details concerning the 
expression of the Jacobian matrix $\partial \mathbf{f} / \partial \mathbf{x}$ and of the gradient vector $\partial \mathbf{h} / \partial \mathbf{x}$ are provided in Appendix B.

The proposed observer has been validated both by means of simulations and through experimental data obtained in a pilot-scale freeze-dryer. In the first case the detailed mono-dimensional model developed by Velardi and Barresi (2008) has been used as a source of simulated experimental data. We remind that this model is fully transient and takes into account the effect of accumulation in the vial glass. The main parameters used in the simulations are given in Table 1. The detailed model has been used for simulations in four different conditions, corresponding to the couples of values of $K_{v}$ and $k_{1}$ reported in Table 2, thus obtaining the set of simulated measured values of the temperature at the bottom of the product. The sample thickness has been taken equal to $20 \mathrm{~mm}$.

Figure 2 shows the observer predictions compared to the simulated temperature at the interface and at the bottom of the product; in Figure 3 the time evolution of the moving front position, calculated from Eq. (11), is plotted. The agreement between the estimation and the real original values is generally good, except for the first part of the drying period, where the performance of the observer are poorer.

Poor initial performances are mainly related to the goodness of the initial approximation of the state of the system, and in part are due to the simplifications introduced by the reduced model. In fact, it should be pointed out that the observer was developed starting from a model that, among the other simplifications, does not take into account the accumulation term in the product; this can play an important role in the first part of the drying cycle, when the temperature decreases due to sublimation at the very low pressure used in simulations and the heat supplied from the shelf is not able to balance the temperature diminution. When the transient effects are no longer important and the estimated dynamics approaches the actual one, the observer can provide a good estimate of the temperature at the interface and of the front position as well all along the rest of the process, giving practically coincident final drying times.

Concerning the parameters $K_{v}$ and $k_{1}$, some considerations can be made by looking at Figure 4, that shows the estimates of the two parameters along the drying cycle. Comparing the values of Table 2 with the estimated ones, it comes out that the effective diffusivity $k_{1}$ is well estimated in all the four cases considered, except for the 
initial part of the drying cycle. Instead, the heat transfer coefficient $K_{v}$ is slightly overestimated by $15-20 \%$. This is due to the simplifications introduced in developing the simplified model: in fact, when considering the complete transient model, the effect of heat transfer in the vial glass is taken into account; thus, the energy coming from the shelf is provided to the product mainly at the bottom of the sample, but to some extent is transferred to the product from the vial sides too as a consequence of conduction through the glass. In the simplified model the presence of the vial is not taken into account and the energy coming from the shelf reaches the product only through the bottom. This results in an effective value of $K_{v}$, higher than the actual one, weighing up the additional heat input due to heat transfer from the vial sides. The way to calculate this effective $K_{v}$ has been shown in Velardi and Barresi (2008). It must be evidenced that the estimated effective values agree with the expected ones, calculated as said above; in addition, the fact that the observer estimates an effective $K_{v}$ is not a limitation in model-based control applications, because in this case a simplified model is generally adopted, that uses this effective coefficient.

Figure 5 shows the predictions of the observer when a noisy measurement of $T_{B}$ is simulated. For this purpose, a normal white noise characterised by a standard deviation of $1 \mathrm{~K}$ was added to the output signal simulated through Eq. (3). It can be seen that the interface temperature (l.h.s) continues to be well estimated, while a different behaviour is observed concerning the position of the ice front (r.h.s). The noise of the measure is in this case amplified, making the $H$ provided by the EKF rather noisy, even if the global trend of the curve is followed.

As discussed before, the tuning of the EKF requires to select the terms of the matrix $\Lambda$, which must be a semi-positive definite matrix, and the value of the tuning parameter $r$. A possible choice consists in assuming a diagonal form for $\Lambda$, with the positive diagonal terms to be selected by trial and error. In the case of our system, $\Lambda$ is a $3 \times 3$ matrix and the selection of the three diagonal terms was made carrying out different simulations in the conditions of case 1 of Table 2, while $r$ was chosen equal to 1. The selected $\Lambda$ was then used for the other simulated cases, showing that the observer robustness is good, when a suitable $\Lambda$ has been defined.

Preliminary experimental results obtained in a Telstar Lyobeta25 freeze-dryer (chamber volume $=0.2 \mathrm{~m}^{3}$ ) confirm the goodness of the state estimation and the 
influence of the measurement noise on the evaluation of the front position. The apparatus used is a special prototype that allows to monitor the product temperature and to estimate the process parameters through the DPE method (Velardi et al., 2008; Barresi et al., 2009a). The DPE operating principle is based on the pressure increase measured during the so-called pressure rise test (PRT) timely run during primary drying, isolating for a few seconds the dryer chamber from the condenser. Thus, DPE provides at discrete times average estimations of the state of the system, since all the vials of the batch contribute to the pressure increase.

The product used in the experimental cycles consisted of a $10 \% \mathrm{w} / \mathrm{w}$ sucrose solution, distributed in 636 tubing type vials (internal diameter $=14.25 \mathrm{~mm}$, filling volume $=1 \mathrm{~mL}$ ). Four vials located in different zones of the tray were equipped with a thermocouple placed in contact with the vial bottom. The product, initially frozen at $42^{\circ} \mathrm{C}$, was maintained at a total pressure of $10 \mathrm{~Pa}$ during the primary drying.

It has been observed that the performance of the observer can be improved if it is coupled with the DPE tool. In fact, especially at the start-up, the error of the estimation requires a certain time to converge to zero; but if the values of the interface temperature, the heat transfer coefficient and the mass transfer resistance calculated with the pressure rise test carried out at the beginning of the of primary drying are used to initialise the observer equations, the time requested by the Kalman filter estimation to converge to the actual solution is minimised.

In Figure $6 \mathrm{a}$ the measured bottom temperature is compared with the estimated values of $T_{B}$ and $T_{i}$. It must be pointed out that the temperature of the moving interface can not be physically measured; thus, the performances of the observer were judged comparing the values of the physical measure $T_{B}$ with the corresponding estimation, provided by Eq. (20), verifying that the dynamics of the measured variable was retrieved satisfactorily.

As anticipated in the Introduction, the state estimation obtained with the method presented in this paper is limited to a single vial. Nevertheless, several vials of the batch can be monitored. Figure $6 \mathrm{~b}$ shows the evolution of the interface temperature estimated in four different vials, compared with the batch average front temperature determined through the DPE method described above. The figure shows that the results of the two methods are consistent, and highlights at the same time the difference in the type of information that can be obtained. The use of several observers can allow the estimation 
of the variance of the batch; in fact, ice nucleation is a stochastic process, and thus different ice structures and different sublimation rates can results. In addition, the effect of temperature differences in the shelf, and eventually a different contribution from wall radiation, can results in different thermal histories for vials in different positions [see for example Barresi et al. (2008a) and Pisano et al. (2008), for an experimental evidence of this]. In this case vial \#2 has a behaviour very close to the average of the batch; this is reasonable as it was placed in the centre of the tray and, apart for intra-batch intrinsic variability, this makes it the most representative for the average batch. This is confirmed in Figure $6 \mathrm{~d}$, where the moving front positions obtained with the two methods are compared and good agreement can be observed too.

The estimation of the front position deserves some additional comments concerning the effect of the noise in the temperature measurements; the simulation work had already highlighted this problem, evidencing that is was affecting more seriously the estimation of the front position, while the interface temperature is only slightly affected (see Fig. 5). The experimental results confirmed this conclusion, evidencing that the noise was amplified up to the point that only the general trend of the interface evolution could be catch adopting Eq. (11) for the direct evaluation of the interface position. A much better results was obtained on the other hand calculating the sublimation rate first, using Eq. (12) and the estimated state of the system, and then the position of the interface by integrating it over time; the results shown in Fig. $6 \mathrm{~d}$ have been obtained by this way.

Finally, Figure 6c shows the temperature profile of the frozen product in the four monitored vials, taken after about $1.5 \mathrm{~h}$ of drying. In fact, it must be pointed out that the proposed observer is able to estimate the entire temperature profile of the product in the vial and not only the temperature in a particular point, as commonly obtained using a thermocouple.

As concerns the value of the other two state variables, the heat and mass transport coefficients, an almost constant value $K_{v}=7 \mathrm{~J} \mathrm{~m}^{-2} \mathrm{~s}^{-1} \mathrm{~K}^{-1}$ is estimated by the EKF, which is consistent with that calculated by means of literature correlations (Pikal et al., 1984); the relatively low value depends on the fact that the product was placed on a tray, that strongly increases the resistance to heat transfer. An higher variability through the monitored vials is observed in the estimated value of mass diffusivity $k_{1}$ : in particular a 
$1.1 \pm 0.3 \cdot 10^{-3} \mathrm{~m}^{2} \mathrm{~s}^{-1}$ average value is obtained for vials \#1 to \#3, while an higher value is obtained for vial \#4 $\left(7.2 \cdot 10^{-3} \mathrm{~m}^{2} \mathrm{~s}^{-1}\right)$. The values obtained are within the range generally reported in literature, and the results evidence a large intra-batch variance, that can have also been favoured by inserting the thermocouple in the product; in fact, during freezing the thermocouple can favour nucleation at lower supercooling, generating larger ice crystal and thus a final matrix with higher conductivity. It is also possible that some compensation between the parameters occurs in the optimisation procedure that leads to the state estimation; this is not a very serious drawback for control applications, if a similar model is used for prediction and state estimate.

\section{Conclusions and final remarks}

In-line monitoring of the primary drying phase of a freeze-drying process in vial has been addressed in this paper. It has been pointed out that the most important parameters to be monitored during primary drying are the temperature of the product, in order to avoid collapse, and the position of the moving front of sublimation, that gives the progress of the primary drying phase. The use of a completely predictive approach through mathematical simulation may not be feasible, since mathematical models of freeze-drying need many parameters, primarily the values of the heat and mass transfer coefficients, that often are not known a priori. Thus, from the simplified model showed in the first part of the work a non-linear observer was synthesised, capable to infer inline the evolution of the moving front temperature and position, without any knowledge of the heat and mass transfer related parameters. The performances of the developed observer, based on the Extended Kalman Filter concept, were validated with a detailed mono-dimensional model and tested with experimental data.

The proposed observer, based on the single point measure of the product temperature at the bottom of the frozen layer, has proven to be able to estimate the state of the system even starting from not perfectly known initial conditions. To this end, it is important to note that the EKF is an intrinsically time-variant non-linear estimator for which there is generally no guarantee to work properly like in the linear case. Nevertheless the filter works well for many application, as for the one presented in this paper, once a proper tuning of the observer has been made.

Differently from methods based on PRT, that provide an average estimation of the 
state of the system, the estimations of the developed EKF are limited to a single vial. Nonetheless, several vials placed in different position in the vacuum chamber could be monitored contemporaneously and independently to allow the evaluation of the heterogeneity of the batch.

Poor process control is a major limitation of freeze-drying where control actions are often based on empirical laws and data collected off-line. Besides monitoring, the method proposed in this paper can be employed to realize an efficient control of the process. In fact, the parameters estimated by the observer could be used to close the loop, enabling an in-line control system capable to manipulate the temperature of the heating shelf in order to maintain the product at a safe temperature level, avoiding product denaturation. Examples of possible application, also in conjunction with the PRT methods, and in particular with the DPE method, are given in Fissore et al. (2008) and Barresi et al. (2009b). It can be noted that coupling with DPE could be particularly advantageous even just for initialization; in fact, it has been shown experimentally that this can guarantee faster convergence to the exact solution, and thus may significantly improve performance and convergence of the observer.

The use of this type of observer for industrial applications is potentially very interesting; in fact it is possible to realize a wireless system to measure the temperature of several vials and send the signal in real-time to an acquisition system placed outside the freeze-dryer (Barresi et al., 2008b, 2009b; Fissore et al., 2009). Thus, this apparatus is suitable to be employed even in very large industrial freeze-dryers equipped with automatic loading and unloading systems.

\section{Acknowledgement}

The authors would like to acknowledge E.U. for financial support in the framework of the research project LYO-PRO: Optimization and control of the freeze-drying of pharmaceutical proteins (GROWTH Project GRD1-2001-40259-RTD).

The authors like to thank Dr R. Pisano for partecipating to the experimental validation of the observer and Dr. D. Fissore (Politecnico of Torino) for the contribution to the revision of the manuscript. 


\section{Appendix A - EKF generalities and convergence conditions.}

A dynamic system is here defined by the following set of differential equations:

$$
\dot{\mathbf{x}}(t)=\mathbf{f}(\mathbf{x}(t), \mathbf{u}(t))+\mathbf{v}(t)
$$

where $\mathbf{x} \in \mathbb{R}^{n}$ is the unknown state of the system, $\mathbf{u} \in \mathbb{R}^{m}$ is a known input signal, $\mathbf{f}$ is an application of $\mathbb{R}^{n} \times \mathbb{R}^{m}$ in $\mathbb{R}^{n}$ giving the derivatives of the state depending on the state itself and on the control law $\mathbf{u}$ applied to the system, and $\mathbf{v}$ is a noise on the state.

In order to build an observer for a given system we need to add to the system (A1) some information concerning the physically measured variables:

$\mathbf{y}(t)=\mathbf{h}(\mathbf{x}(t), \mathbf{u}(t))+\mathbf{w}(t)$

where the components of the vector $\mathbf{y} \in \mathbb{R}^{q}$ are the output signal, and $\mathbf{w}$ is a noise on the output.

The Kalman Filter is essentially a set of mathematical equations that implement a predictor-corrector type estimator that is optimal in the sense that it minimizes the estimated error covariance when some presumed conditions are met; the extended Kalman filter (EKF) is based on the linearization of the non-linear functions of the process. We recall here the condition which guaranties the convergence of the filter. The EKF is given by the following dynamical system:

$$
\begin{aligned}
& \dot{\hat{\mathbf{x}}}(t)=\mathbf{f}(\hat{\mathbf{x}}(t), \mathbf{u}(t))-r \mathbf{S}^{-1}(t)\left(\left.\frac{\partial \mathbf{h}}{\partial \mathbf{x}}\right|_{\hat{\mathbf{x}}, u}\right)^{T}\left(\mathbf{h}(\hat{\mathbf{x}}(t), \mathbf{u}(t))-\mathbf{y}_{\text {meas }}(t)\right)+\mathbf{v}(t) \\
& \dot{\mathbf{S}}(t)=-\mathbf{S}(t) \Lambda \mathbf{S}(t)-\left(\left.\frac{\partial \mathbf{f}}{\partial \mathbf{x}}\right|_{\hat{\mathbf{x}}, \mathbf{u}}\right)^{T} \mathbf{S}(t)-\left.\mathbf{S}(t) \frac{\partial \mathbf{f}}{\partial \mathbf{x}}\right|_{\hat{\mathbf{x}}, \mathbf{u}}+\left.r\left(\left.\frac{\partial \mathbf{h}}{\partial \mathbf{x}}\right|_{\hat{\mathbf{x}}, \mathbf{u}}\right)^{T} \frac{\partial \mathbf{h}}{\partial \mathbf{x}}\right|_{\hat{\mathbf{x}}, \mathbf{u}}
\end{aligned}
$$

where $\mathbf{y}_{\text {meas }}(t)$ is the physical on-line output measurement, $\Lambda$ is a symmetric positive definite (SDP) matrix and $r>0$ is a tuning parameter. $\mathbf{S}(0)$ is a SDP matrix. The hypothesis concerning the process is that the unknown state of the process, $\mathbf{x}(t)$, and the physical output, $\mathbf{y}_{\text {meas }}(t)$, satisfy the equations of the prediction model given by Eqs. (A1)-(A2). Set $\mathbf{e}(t)=\hat{\mathbf{x}}(t)-\mathbf{x}(t)$ to be the estimation error, the first order Taylor expansion yields: 
$\dot{\mathbf{e}}(t)=\left.\frac{\partial \mathbf{f}}{\partial \mathbf{x}}\right|_{\hat{\mathbf{x}}, u}-\left.r \mathbf{S}^{-1}(t)\left(\left.\frac{\partial \mathbf{h}}{\partial \mathbf{x}}\right|_{\hat{\mathbf{x}}, u}\right)^{T} \frac{\partial \mathbf{h}}{\partial \mathbf{x}}\right|_{\hat{\mathbf{x}}, u} \mathbf{e}(t)+\boldsymbol{\varphi}\left(\|\mathbf{e}(t)\|^{2}\right)+\boldsymbol{\Psi}(\mathbf{v}(t), \mathbf{w}(t))$

where $\frac{\varphi\left(\|\mathbf{e}(t)\|^{2}\right)}{\|\mathbf{e}(t)\|^{2}}$ and $\frac{\Psi(\mathbf{v}, \mathbf{w})}{\|\mathbf{v}\|+\|\mathbf{w}\|}$ are bounded functions on any bounded set of $\mathbf{e}, \mathbf{v}$, and $\mathbf{w}$.

The hypothesis given by Kalman and Bucy (1961) for time variant linear systems consists in assuming that the following artificial linearized system along $(\hat{\mathbf{x}}(t), \mathbf{u}(t))$ :

$$
\begin{aligned}
& \dot{\boldsymbol{\xi}}(t)=\left.\frac{\partial \mathbf{f}}{\partial \mathbf{x}}\right|_{\hat{\mathbf{x}}, u} \boldsymbol{\xi}(t) \\
& \mathbf{Y}_{\xi}(t)=\left.\frac{\partial \mathbf{h}}{\partial \mathbf{x}}\right|_{\hat{\mathbf{x}}, u} \boldsymbol{\xi}(t)
\end{aligned}
$$

is completely uniformly observable. Under this hypothesis, there exist two constants $c_{1}>0$ and $\quad c_{2}>0$ such that $c_{1}\|\mathbf{e}(t)\|^{2} \leq \mathbf{e}^{T} \mathbf{S}(t) \mathbf{e} \leq c_{2}\|\mathbf{e}(t)\|^{2}$. Set $\mathbf{V}(t)=\mathbf{e}^{T}(t) \mathbf{S}(t) \mathbf{e}(t)$, after some calculations, we can show that there exist some constants $c>0, c^{\prime}>0$, and $c^{\prime \prime}>0$ such that:

$$
\begin{aligned}
\dot{\mathbf{V}}(t) \leq & \mathbf{e}^{T}(t) \mathbf{S}(t) \Lambda \mathbf{S}(t) \mathbf{e}(t)+\mathbf{e}^{T}(t) \mathbf{S}(t)\left[\boldsymbol{\varphi}\left(\|\mathbf{e}(t)\|^{2}\right)+\boldsymbol{\Psi}(\mathbf{v}(t), \mathbf{w}(t))\right] \\
\leq & -c \mathbf{e}^{T}(t) \mathbf{S}(t) \mathbf{S}(t) \mathbf{e}(t)+c^{\prime}\|\mathbf{e}(t)\| \mathbf{e}^{T}(t) \mathbf{S}(t) \mathbf{S}(t) \mathbf{e}(t) \\
& +c^{\prime \prime} \sqrt{\mathbf{e}^{T}(t) \mathbf{S}(t) \mathbf{S}(t) \mathbf{e}(t)} \sqrt{\|\mathbf{v}(t)\|+\|\mathbf{w}(t)\|}
\end{aligned}
$$

Hence, if $\mathbf{e}(0)$ is in some local domain of the origin, and if $\|\mathbf{v}(t)\|=\|\mathbf{w}(t)\|=0$, then $\mathbf{e}(t)$ exponentially converges to 0 ; if $\|\mathbf{v}(t)\|+\|\mathbf{w}(t)\| \neq 0$, then $\mathbf{e}(t)<\varepsilon$, where $\varepsilon$ is a constant which depends on the upper bounds of $\mathbf{v}$ and $\mathbf{w}$. If $\mathbf{v}$ and $\mathbf{w}$ are small, so is for $\varepsilon$.

\section{Appendix B - Details of Jacobian and gradients calculations.}

The Jacobian matrix $\partial \mathbf{f} / \partial \mathbf{x}$ and the gradient vector $\partial h / \partial \mathbf{x}$, needed to compute the equations of the dynamic system (19)-(22), have the following form:

$$
\begin{aligned}
& \left.\frac{\partial h}{\partial \mathbf{x}}\right|_{\hat{\mathbf{x}}, u}=\left(\begin{array}{ccc}
\frac{\partial h}{\partial x_{1}} & \frac{\partial h}{\partial x_{2}} & \frac{\partial h}{\partial x_{3}}
\end{array}\right)_{\hat{\mathbf{x}}, u} \\
& \left.\frac{\partial \mathbf{f}}{\partial \mathbf{x}}\right|_{\hat{\mathbf{x}}, u}=\left[\begin{array}{ccc}
\frac{\partial f_{1}}{\partial x_{1}} & \frac{\partial f_{1}}{\partial x_{2}} & \frac{\partial f_{1}}{\partial x_{3}} \\
0 & 0 & 0 \\
0 & 0 & 0
\end{array}\right]_{\hat{\mathbf{x}}, u}
\end{aligned}
$$

The components of the gradient of $h(\mathbf{x}, u)$ can be calculated by derivation of equation 
(20), obtaining:

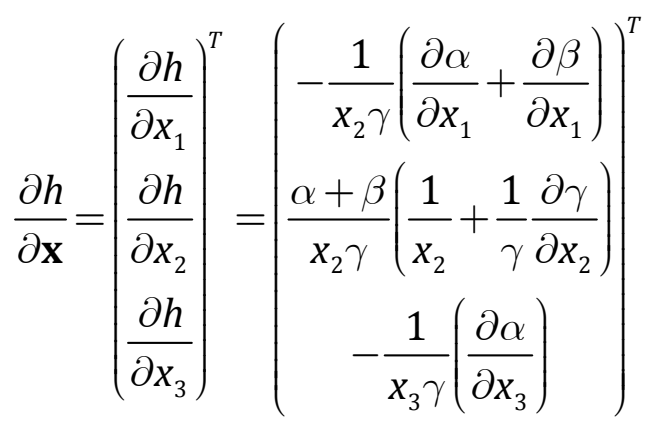

while the components of the first row of the Jacobian of $\mathbf{f}(\mathbf{x}, u)$ are derived from equation (19), getting:

$$
\begin{aligned}
\frac{\partial f_{1}}{\partial x_{1}}= & \delta\left(\frac{\alpha+\beta}{\gamma}\right)^{2} \frac{1}{\beta \frac{\partial \alpha}{\partial x_{1}}-\frac{d \beta}{d x_{1}} \alpha}\left[3\left(\frac{\partial \alpha}{\partial x_{1}}+\frac{d \beta}{d x_{1}}\right)+(\alpha+\beta) \frac{\beta \frac{\partial^{2} \alpha}{\partial x_{1}^{2}}-\frac{\partial^{2} \beta}{\partial x_{1}^{2}} \alpha}{\beta \frac{\partial \alpha}{\partial x_{1}}-\frac{d \beta}{d x_{1}} \alpha}\right] \\
& +\frac{d \beta}{d u} \frac{d u}{d t} \frac{1}{\beta \frac{\partial \alpha}{\partial x_{1}}-\frac{d \beta}{d x_{1}} \alpha}\left[\frac{\partial \alpha}{\partial x_{1}}-\alpha \frac{\beta \frac{\partial^{2} \alpha}{\partial x_{1}^{2}}-\frac{\partial^{2} \beta}{\partial x_{1}^{2}} \alpha}{\beta \frac{\partial \alpha}{\partial x_{1}}-\frac{d \beta}{d x_{1}} \alpha}\right] \\
\frac{\partial f_{1}}{\partial x_{2}}= & \delta\left(\frac{\alpha+\beta}{\gamma}\right)^{2} \frac{1}{\beta \frac{\partial \alpha}{\partial x_{1}}-\frac{d \beta}{d x_{1}} \alpha}\left[\frac{-2(\alpha+\beta)}{\gamma} \frac{\partial \gamma}{\partial x_{2}}\right] \\
\frac{\partial f_{1}}{\partial x_{3}}= & \delta\left(\frac{\alpha+\beta}{\gamma}\right)^{2} \frac{1}{\beta \frac{\partial \alpha}{\partial x_{1}}-\frac{d \beta}{d x_{1}} \alpha}\left[3 \frac{\partial \alpha}{\partial x_{3}}+(\alpha+\beta) \frac{\frac{\partial \beta}{\partial x_{1}} \frac{\partial \alpha}{\partial x_{3}}-\beta \frac{\partial^{2} \alpha}{\partial x_{1} \partial x_{3}}}{\beta \frac{\partial \alpha}{\partial x_{1}}-\frac{d \beta}{d x_{1}} \alpha}\right] \\
& +\frac{d \beta}{d u} \frac{d u}{d t} \frac{1}{\beta \frac{\partial \alpha}{\partial x_{1}}-\frac{d \beta}{d x_{1}} \alpha}\left[\frac{\partial \alpha}{\partial x_{3}}-\alpha \frac{\frac{\partial \beta}{\partial x_{1}} \frac{\partial \alpha}{\partial x_{3}}-\beta \frac{\partial^{2} \alpha}{\partial x_{1} \partial x_{3}}}{\beta \frac{\partial \alpha}{\partial x_{1}}-\frac{d \beta}{d x_{1}} \alpha}\right]
\end{aligned}
$$

where the derivatives of the functions $\alpha\left(x_{1}, x_{3}\right), \beta\left(x_{1}, u\right)$, and $\gamma\left(x_{2}\right)$ can be determined by the trivial derivation of Eq. (12)-(14):

$$
\begin{aligned}
& \frac{\partial \alpha}{\partial x_{1}}=\frac{\Delta H_{s} M}{R} \frac{x_{3}}{x_{1}}\left(-\frac{p_{\mathrm{w}, \mathrm{i}}\left(x_{1}\right)-p_{\mathrm{c}}}{x_{1}}+\frac{d p_{\mathrm{w}, \mathrm{i}}}{d x_{1}}\right) \\
& \frac{\partial \alpha}{\partial x_{3}}=\frac{\Delta H_{s} M}{R} \frac{p_{\mathrm{w}, \mathrm{i}}\left(x_{1}\right)-p_{\mathrm{c}}}{x_{1}} \\
& \frac{\partial^{2} \alpha}{\partial x_{1}^{2}}=-\frac{2}{x_{1}} \frac{\partial \alpha}{\partial x_{1}}+\frac{\Delta H_{s} M}{R} \frac{x_{3}}{x_{1}} \frac{d^{2} p_{\mathrm{w}, \mathrm{i}}}{d x_{1}^{2}} \\
& \frac{\partial^{2} \alpha}{\partial x_{1} \partial x_{3}}=\frac{1}{x_{3}} \frac{\partial \alpha}{\partial x_{1}}
\end{aligned}
$$




$$
\begin{aligned}
& \frac{\partial \beta}{\partial x_{1}}=-k_{\mathrm{II}} \\
& \frac{\partial \beta}{\partial u}=k_{\mathrm{II}} \\
& \frac{\partial^{2} \beta}{\partial x_{1}^{2}}=0 \\
& \frac{\partial \gamma}{\partial x_{2}}=-\frac{k_{\mathrm{II}}}{x_{2}^{2}}
\end{aligned}
$$




\section{Notation}

$\mathbf{e}(t) \quad$ error of estimation of the observer

f vectorial function giving the derivatives of the state

h vector of equations giving the state space equations of the measures

H moving front position, $\mathrm{m}$

$\Delta H_{s} \quad$ enthalpy of sublimation, $\mathrm{J} \mathrm{kg}^{-1}$

$k \quad$ thermal conductivity, $\mathrm{J} \mathrm{m}^{-1} \mathrm{~s}^{-1} \mathrm{~K}^{-1}$

$k_{1} \quad$ effective diffusivity coefficient, $\mathrm{m}^{2} \mathrm{~s}^{-1}$

$\mathbf{K}(t) \quad$ observer gain

$K_{\mathrm{v}} \quad$ overall heat transfer coefficient, $\mathrm{J} \mathrm{m}^{-2} \mathrm{~s}^{-1} \mathrm{~K}^{-1}$

$L \quad$ total product thickness, $\mathrm{m}$

$M \quad$ molecular weight, $\mathrm{kmol} \mathrm{kg}^{-1}$

$p \quad$ pressure, $\mathrm{Pa}$

$R \quad$ ideal gas constant, $\mathrm{J} \mathrm{kmol}^{-1} \mathrm{~K}$

$r \quad$ tuning parameter

$\mathbf{S}(t) \quad$ matrix giving the solution of the dynamic Riccati equation

$t \quad$ time, $\mathrm{s}$

$T \quad$ Temperature, $\mathrm{K}$

$T_{B} \quad$ frozen layer temperature at $z=L, \mathrm{~K}$

$\mathbf{u} \quad$ vector of the control variables

$\mathbf{v}(t), \mathbf{w}(t)$ white noises

x state space vector

y vector of the measured outputs of the system

$z \quad$ axial coordinate, $\mathrm{m}$

Greeks

$\alpha, \beta, \gamma, \delta \quad$ variables defined by equations (12)-(15)

$\Lambda \quad$ matrix of tuning parameters for the Kalman observer 
$\varrho \quad$ mass density, $\mathrm{kg} \mathrm{m}^{-3}$

\section{Subscripts}

$\begin{array}{ll}\text { I } & \text { layer I, dried layer } \\ \text { II } & \text { layer II, frozen layer } \\ c & \text { chamber } \\ e & \text { effective } \\ i & \text { interface } \\ \text { meas } & \text { measured } \\ \text { shelf } & \text { heating shelf } \\ w & \text { water vapour }\end{array}$

Superscripts

$\wedge \quad$ observer estimate

first time derivative

Abbreviations

DPE Dynamic Parameters Estimation

EKF Extended Kalman Filter

MTM Manometric Temperature Measurement

PRT Pressure Rise Test

SDP Semi Definite Positive 


\section{References}

Alamir, M., \& Calvillo-Corona, L. A. (2002). Further results on nonlinear recedinghorizon observers. IEEE Transactions on Automatic Control, 47(7), 11841188.

Alvarez, A., \& Simutis R. (2004) Application of Kalman filter algorithms in a GMC control strategy for fed-batch cultivation process. Informacines Technologijos ir Valdymas, 1 (30), 7-12.

Barresi, A. A., Pisano, R., Rasetto, V., Fissore, D., Marchisio, D. L., \& Galan M. (2008a) Model-based monitoring and controlling of industrial freeze-drying processes. Drying 2008 - Proceedings of the 16th International Drying Symposium (IDS2008) (B. N. Thorat, Ed.), November 9-12, Hyderabad, India, Vol. B, 746-754.

Barresi, A. A., Rasetto, V., Marchisio, D. L., Fissore, D., \& Pisano, R. (2008b). Understanding, monitoring and control the freeze drying process by means of model based tools and Computational Fluid Dynamics. Presented at International Society for Pharmaceutical Engineering (ISPE) Conference on Lyophilisation - Applying a Science and Risk-based Approach, December 3-4 2008, Barcelona, Spain.

Barresi, A. A., Pisano, R., Fissore, D., Rasetto, V., Velardi, S., Vallan, A., Parvis, M., \& Galan, M. (2009a). Monitoring of the primary drying of a lyophilization process in vials. Chemical Engineering and Processing, 48(1), 408-423.

Barresi, A. A., Velardi, S. A., Pisano, R., Rasetto, V., Vallan, A., \& Galan, M. (2009b). In-line control of the lyophilization process. A gentle PAT approach using software sensors. International Journal of Refrigeration. DOI:10.1016/j.ijrefrig.2008.10.012, in press.

Bentes Freire, F., \& Giudici, R. (2004) Temperature oscillation calorimetry by means of a Kalman-like observer: The joint estimation of $\mathrm{Qr}$ and UA in a stirred tank polymerization reactor. Macromolecular Symposia, 206, 15-28.

Cutler, C., Morshedi, A., \& Haydel, J. (1983). An industrial perspective on advanced control. AICHE Annual meeting, Washington, DC, U.S.A..

Dimitratos, J., Georgakis, C., El-Aasser, M., \& Klein, A. (1991) An experimental study 
of adaptive Kalman filtering in emulsion copolymerization. Chemical Engineering Science, 46, 3203-3218.

Fissore, D., Velardi, S. A., \& Barresi, A. A. (2008). In-line control of a freeze-drying process in vials. Drying Technology, 26, 685-694.

Fissore, D., Pisano, R., Rasetto, V., Marchisio, D.L., Barresi, A. A., Vallan A., \& Corbellini, S., 2009, Applying Process Analytical Technology (PAT) to the lyophilization process. Chem. Today/Chimica Oggi, in press.

Hammouri, H., \& Gauthier, J. P. (1992). Global time-varying linearization up to output injection. SIAM Journal on Control and Optimization, 30(6), 12951310.

Kalman, R. E., \& Bucy, R. S. (1961). New results in linear filtering and prediction theory. ASME Journal of Basic Engineering, 83, 95-108.

Kang, W. (2006). Moving Horizon Numerical Observers of Nonlinear Control Systems, IEEE Transactions on Automatic Control, 51(2), 344-350.

Krämer, S., Gesthuisen, R., Engell, S., \& Asua, J. M. (2003), Simultaneous estimation of heat transfer coefficient and reaction heat in semi-batch processes. Proceedings of the American Control Conference, IEEE, June 4-6, Denver, Colorado, Vol. 3, 1980-1981.

Krener, A. J., \& Isidori, A. (1983). Linearization by output injection and nonlinear observers. Systems Control Letters, 3(1), 47-52.

Pikal, M. J., Roy, M. L., Shah, S. (1984). Mass and heat transfer in vial freeze-drying of pharmaceuticals: role of the vial. Journal of pharmaceutical sciences, 73(9), 1224-1237.

Pisano, R., Rasetto, V., Petitti, M., Barresi, A. A., \& Vallan, A. (2008). Modelling and experimental investigation of radiation effects in a freeze-drying process. Abstracts of 5th Chemical Engineering Conference for Collaborative Research in Eastern Mediterranean Countries EMCC5 (F. Scura, M. Liberti, G. Barbieri, E. Drioli, Eds.), ITM-CNR, May 24-29, Cetraro, Italy, 394-398.

Qin, S. J., \& Badgwell, T. A. (1997) An overview of industrial model predictive control technology. In: J. C. Kantor, C. E. Garcia, \& B. Carnahan (Eds.), Chemical process control-V, Fifth International Conference on Chemical Process Control, CACHE and AICHE, pp. 232-256. 
Richalet, J. (1993) Industrial applications of Model-Based Predictive Control. Automatica, 29, 1251-1274.

Velardi, S. A. Freeze-drying: Modelling, monitoring and control. PhD Thesis, Politecnico of Torino (Italy), 2004.

Velardi, S. A., \& Barresi, A. A. (2008). Development of simplified models for the freeze-drying process and investigation of the optimal operating conditions. Chemical Engineering Research and Design, 86, 9-22.

Velardi, S. A., Rasetto, V., \& Barresi A. A. (2008). Dynamic Parameters Estimation method: advanced Manometric Temperature Measurement approach for freeze-drying monitoring of pharmaceutical solutions. Industrial Engineering Chemistry Research, 47(21), 8445-8557. 


\section{List of Tables}

Table $1 \quad$ Values of the parameters used in the simulations.

Table 2 Values of the transport coefficients used in detailed model simulations. 


\section{List of Figures}

Figure $1 \quad$ Schematic of the vial.

Figure 2 Comparison between true and estimated temperatures for the case studies of Table 2. Solid line $(-)$ ): $T_{i}$ Kalman filter estimation; solid circles $(\bullet): T_{i}$ detailed model; open circles (०): $T_{B}$ detailed model.

Figure 3 Comparison between true and estimated moving front position for the case studies of Table 2. Solid line $(-)$ ): $H$ Kalman filter estimation; solid circles $(\bullet): H$ detailed model.

Figure $4 \quad$ Values of the parameters $K_{v}$ and $k_{i}$ for the case studies of Table 2, estimated by Kalman filter.

Figure 5 Kalman filter predictions when a noisy measure is used. Condition of Table 2, case 1. Left: comparison between true and estimated temperatures. Solid line $(-)$ : $T_{i}$ Kalman filter; solid circles $(\bullet): T_{i}$ detailed model; dashed line $(---): T_{B}$ noisy. Right: moving front position. Solid line $(-)$ ): $H$ Kalman filter; solid circles $(\bullet)$ : $H$ detailed model.

Figure $6 \quad$ Freeze-drying of a $10 \% \mathrm{w} / \mathrm{w}$ sucrose solution at $10 \mathrm{~Pa}$.

(a) Comparison between experimental and estimated temperatures. Solid line (—): estimated $T_{i}$; dashed line $(---)$ : estimated $T_{B}$; open circles (O): experimental $T_{B}$. (b) Solid line (— $)$ : estimated front temperature in four vials placed in different positions on the tray; solid circles $(\bullet): T_{i}$ determined through DPE method. (c) Temperature profile along the frozen product in the vials monitored as for graph (b) at $t=5000 s$. (d) solid line (— ): front position estimated from temperature measurements of graph (a); solid circles $(\bullet)$ : front position determined through DPE. 
Table 1

\begin{tabular}{|c|c|c|}
\hline Parameter & Value & Unit \\
\hline$p$ & 1.08 & $\mathrm{~Pa}$ \\
\hline$T_{\text {shelf }}$ & 263.15 & K \\
\hline$k_{I I}$ & 2.56 & $\mathrm{~W} \mathrm{~m}^{-1} \mathrm{~K}^{-1}$ \\
\hline$\varrho_{I e}$ & 328 & $\mathrm{~kg} \mathrm{~m}^{-3}$ \\
\hline$\varrho_{I I}$ & 1030 & $\mathrm{~kg} \mathrm{~m}^{-3}$ \\
\hline$p\left(T_{i}\right)$ & $\begin{array}{c}\exp [-20.9470(273.156 / T-1) \\
-3.56654 \ln (273.156 / T) \\
+2.0189(1-273.156 / T) \\
-5.0983] \\
\end{array}$ & $\mathrm{Pa}$ \\
\hline$\Delta H_{s}$ & $2687.4 \cdot 10^{-3}$ & $\mathrm{~J} \mathrm{~kg}^{-1}$ \\
\hline$L$ & $2 \cdot 10^{-3}$ & $\mathrm{~m}$ \\
\hline
\end{tabular}


Table 2

\begin{tabular}{ccc}
\hline $\begin{array}{c}\text { Detailed model } \\
\text { simulation }\end{array}$ & $\begin{array}{c}K_{\mathrm{v}} \\
{\left[\mathrm{W} \mathrm{m}^{-2} \mathrm{~K}^{-1}\right]}\end{array}$ & $\begin{array}{c}k_{1}\left(\times 10^{3}\right) \\
{\left[\mathrm{m}^{2} \mathrm{~s}^{-1}\right]}\end{array}$ \\
\hline Case 1 & 29.1 & 2.97 \\
\hline Case 2 & 38.5 & 2.97 \\
\hline Case 3 & 29.1 & 5.94 \\
\hline Case 4 & 38.5 & 5.94 \\
\hline
\end{tabular}


Figure 1

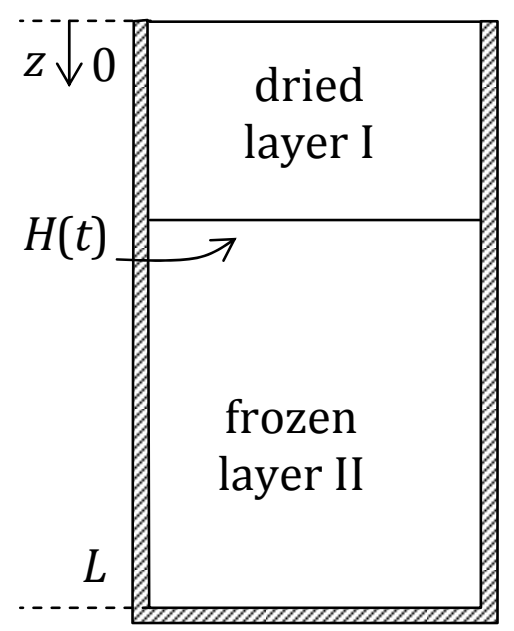


Figure 2
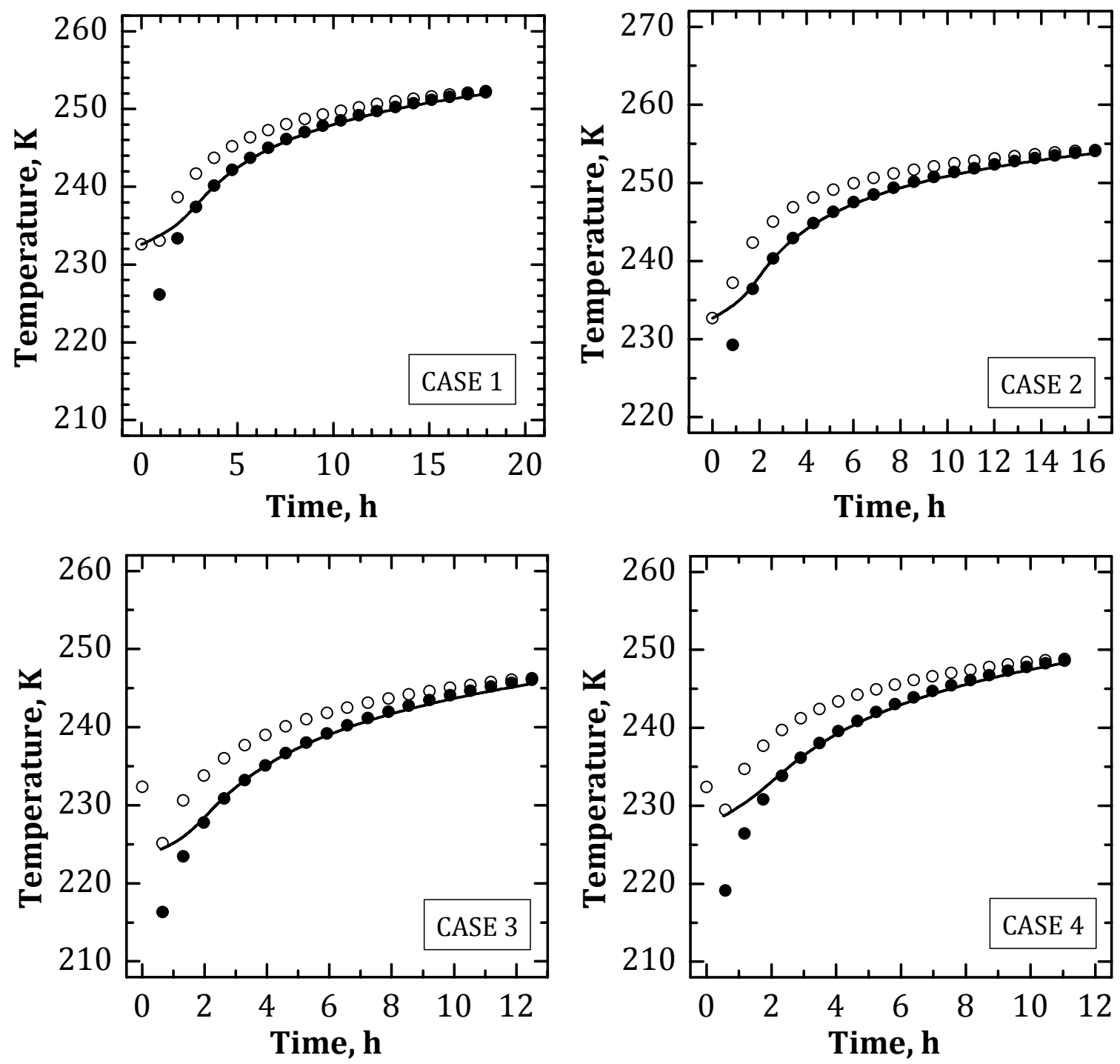
Figure 3
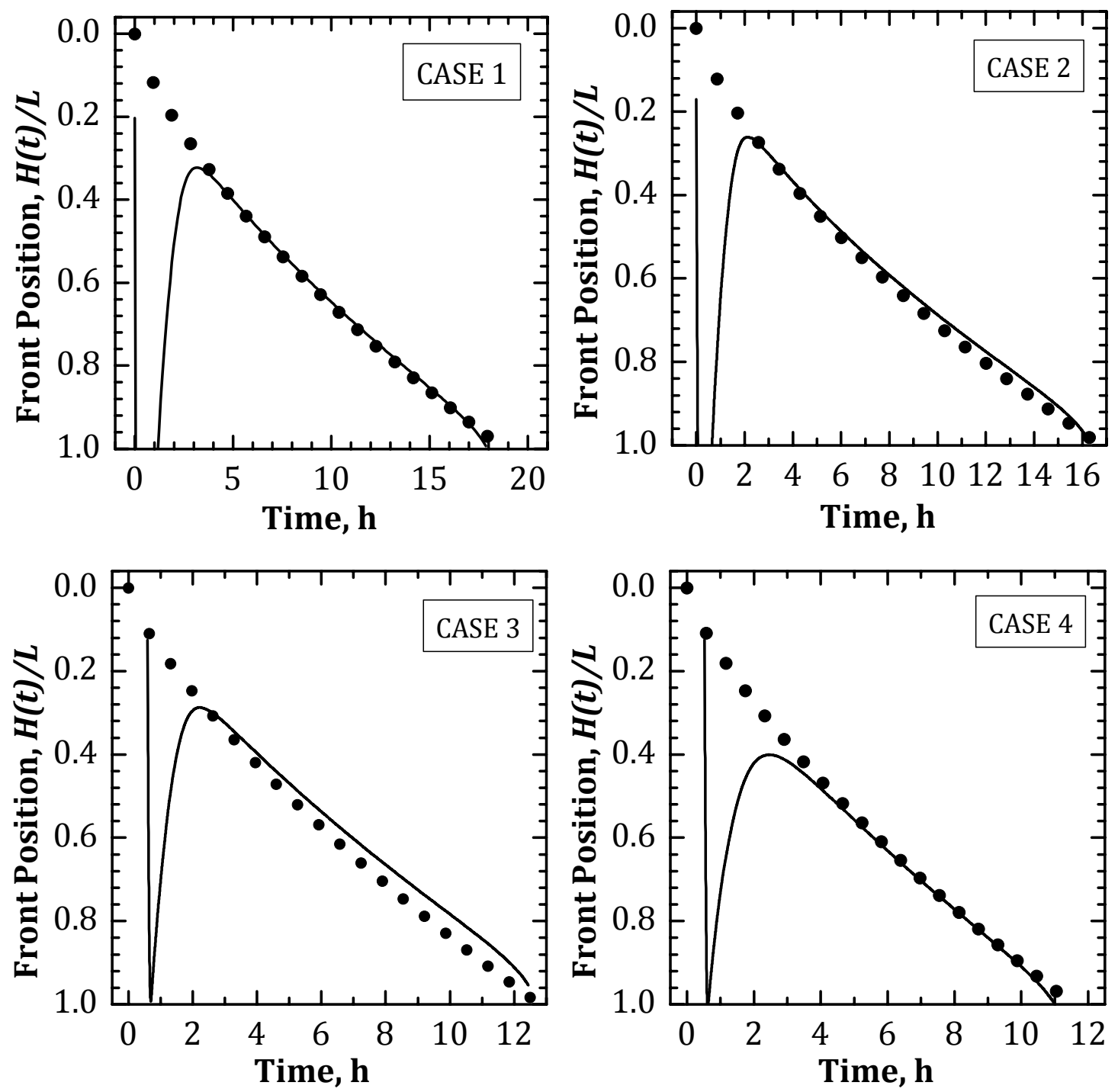
Figure 4
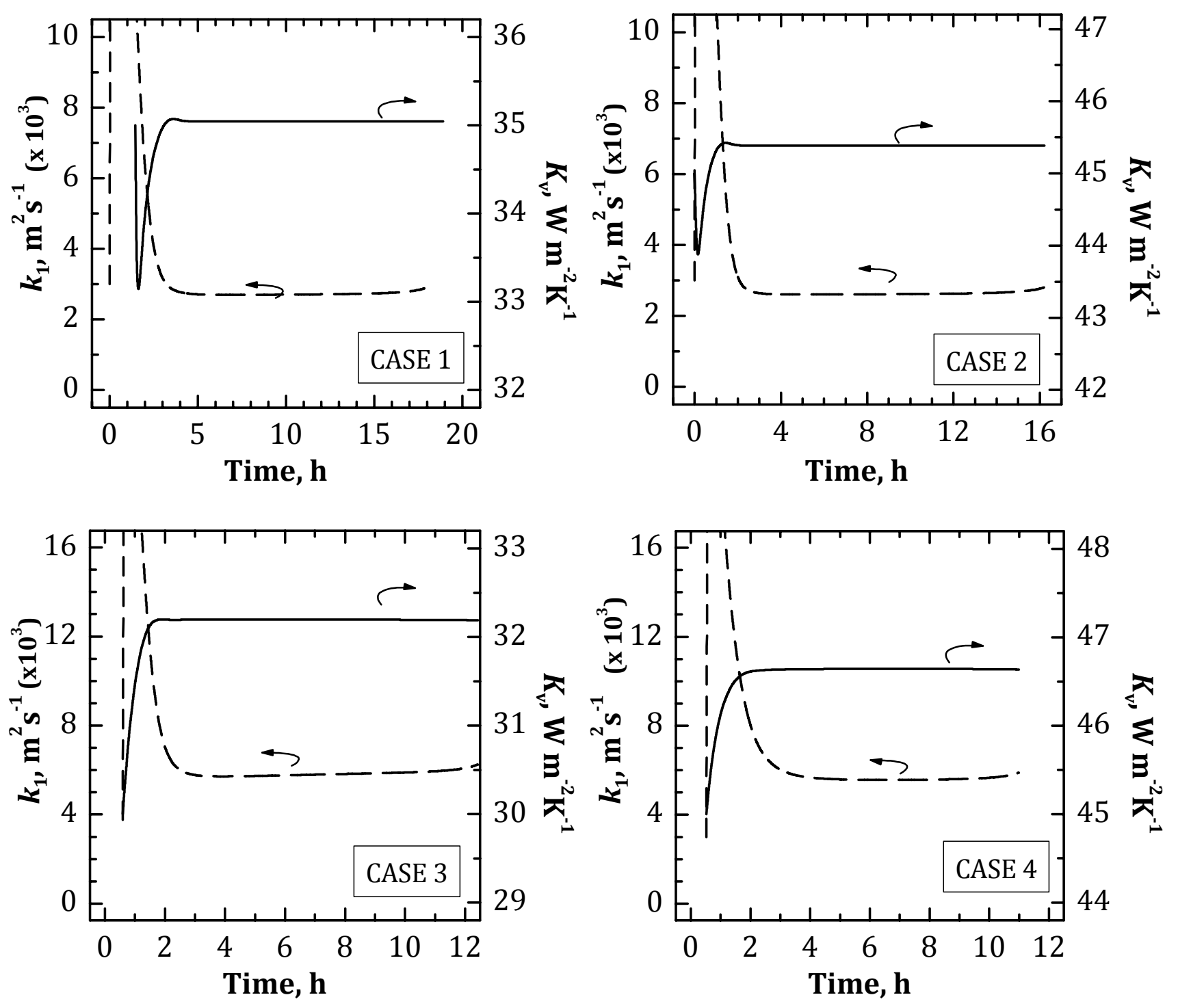
Figure 5
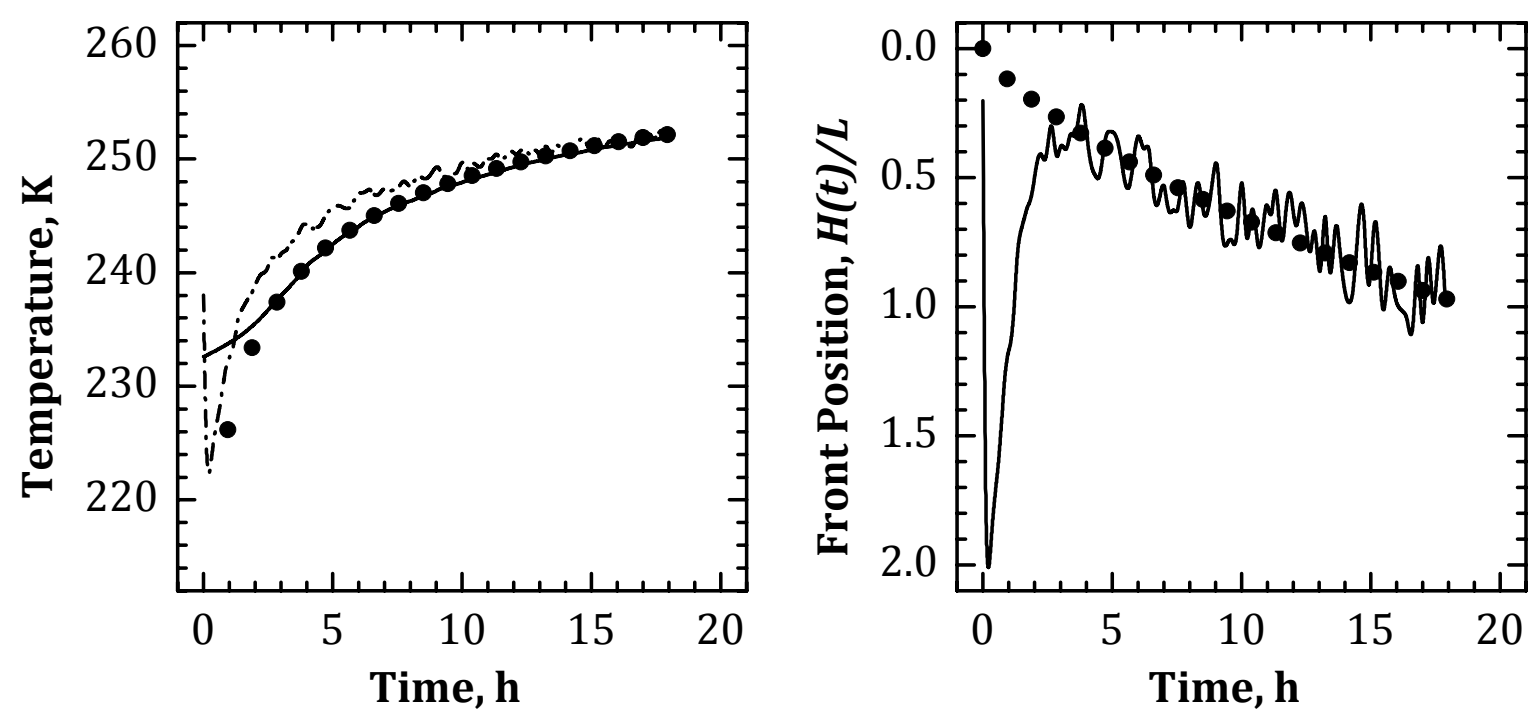
Figure 6
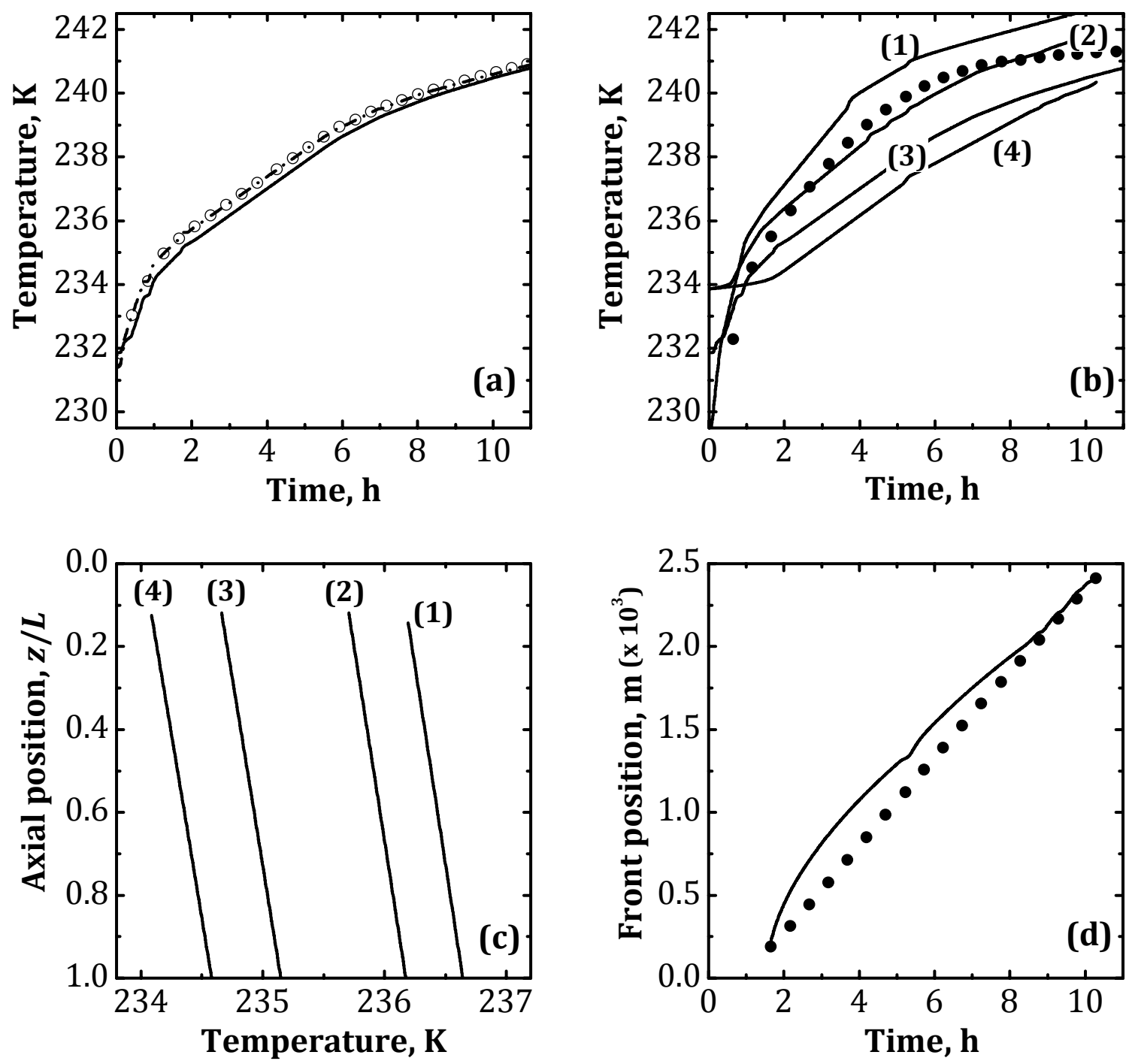